\title{
PARAFUSO DE MASSA LATERAL DO ATLAS PARA FIXAÇÃO DA COLUNA CERVICAL SUPERIOR - RESULTADOS CIRÚRGICOS
}

\author{
LATERAL MASS SCREWS OF THE ATLAS FOR UPPER CERVICAL SPINE FIXATION - \\ SURGICAL RESULTS
}

\section{TORNILLOS DE MASA LATERAL DEL ATLAS PARA LA FIJACIÓN DE LA COLUMNA CERVICAL SUPERIOR - RESULTADOS QUIRÚRGICOS}

Enrico Ghizoni ${ }^{1,2,3}$, Andrel Fernandes Joaquim ${ }^{1,2,3}$, Diogo Valli Andrerle² ${ }^{2}$ Sérgio Raimundo Onofre Cabral ${ }^{3}$

\begin{abstract}
RESUMO
Objetivo: Apresentar os resultados cirúrgicos de uma série de casos de estabilização da coluna cervical superior com o uso de parafusos de massa lateral do atlas. Métodos: Avaliação retrospectiva dos resultados cirúrgicos de pacientes submetidos à estabilização da coluna cervical superior com o uso de parafusos de massa lateral do atlas. Resultados: Seis pacientes foram operados durante o período de janeiro de 2009 a abril de 2010, quatro homens e duas mulheres. Não houve morbidade permanente ou morbidade nessa série de casos. A principal causa de instabilidade atlanto-axial foi trauma e houve apenas um caso de fratura patológica do odontoide por metástase de próstata. A fixação do áxis foi obtida com o uso de três diferentes técnicas de parafusos (pars, pedicular e de lâmina), com igual distribuição entre os pacientes. Conclusão: O uso de parafusos na massa lateral do atlas é uma importante técnica para se obter estabilidade e fusão na coluna cervical superior, e com o conhecimento da anatomia e da técnica cirúrgica, bons resultados podem ser atingidos.
\end{abstract}

Descritores: Atlas cervical; Áxis; Parafusos ósseos; Articulação atlanto-axial; Anatomia;

\begin{abstract}
Objective: To present the surgical results of a case series of upper cervical spine stabilization with the use of lateral mass screws of the atlas. Methods: Retrospective review of the surgical results of patients submitted to upper cervical spine stabilization with the use of lateral mass screws of the atlas. Results: Six patients were operated in the period between January 2009 to April 2010, four men and two women. There was no permanent morbidity or mortality in the presented series. The main cause of atlanto-axial instability was trauma and there was just one case of odontoid pathologic fracture from a prostate metastasis. Axis fixation was achieved with the use of three different screw techniques (pars, pedicle and laminar), with equal distribution among the patients. Conclusions: The use of lateral mass screws of the atlas is an important technique to achieve fusion and stability of the upper cervical spine and with the knowledge of the anatomy and of the surgical technique good results can be achieved.
\end{abstract}

Keywords: Cervical atlas; Axis; Bone screws; Atlanto-axial joint; Anatomy.

\section{RESUMEN}

Objetivos: presentar los resultados quirúrgicos de una serie de casos de estabilización con el uso de tornillos de masa lateral del atlas. Métodos: evaluación retrospectiva de los resultados quirúrgicos de los pacientes sometidos a estabilización de la columna cervical superior con el uso de tornillos de masa lateral del atlas. Resultados: Seis pacientes operados entre enero 2009 y abril 2010 , siendo cuatro hombres y dos mujeres. No hubo mortalidad o morbilidad grave en esta serie. La principal causa de inestabilidad atlantoaxial fue traumática con apenas un caso de fractura patológica del odontoide por una metástasis de tumor de próstata. Fijación del axis fue alcanzada con el uso de tres diferentes técnicas (pars, pedicular, laminar), con dos casos cada una. Conclusión: el uso de tornillos de masa lateral en el atlas es una importante técnica para alcanzar la fusión y la estabilidad de la columna cervical, y con el conocimiento de la anatomía y de la técnica quirúrgica es posible obtenerse excelentes resultados.

Descriptores: Atlas cervical; Axis; Tornillos óseos; Articulación atlantoaxoidea; Anatomía.

\section{INTRODUÇÃO}

O complexo anatômico atlanto-axial é um estrutura anatômica e funcional complexa, composto pelas vértebras de C1 (atlas) e de C2 (áxis), bem como por seus inúmeros ligamentos ${ }^{1}$. Sua arquitetura é importante, permitindo movimentos de flexão, extensão e rotação, bem como inclinação lateral, sendo responsável por mais da metade dos movimentos do crânio em relação a coluna torácica². Inúmeras doenças podem afetar este segmento (trauma, degenerativas, inflamatórias, neoplásicas ou mesmo congênitas), levando a instabilidade com consequente necessidade de fusão para adequada imobilização e estabilidade ${ }^{2}$. O uso de parafusos de massa lateral em C1, inicialmente descrito por Goel e Laheri ${ }^{3}$ é

1. Neurocirurgião - Universidade Estadual de Campinas (UNICAMP) - Campinas - SP - Brasil.

2. Neurocirurgião, Hospital Estadual Carlos Lacaz - Francisco Morato - SP - Brasil.

3. Neurocirurgião do Hospital Santa Tereza - Campinas - SP - Brasil.

Trabalho realizado no Hospital Estadual de Francisco Morato - São Paulo - SP - Brasil.

Correspondência: Rua Antonio Lapa, 280 SL 506 - Cambuí - CEP 13025-240 / Campinas - SP - Brasil - E-mail: ghizonie@gmail.com

Recebido em 16/12/2010, aceito em 22/03/2011. 
uma das melhores técnicas para se alcançar a fusão deste segmento, podendo ser associado com outras formas de fixação occipito cervical, em C2 (parafusos de pars, pedículo, lâmina, ganchos) e na coluna cervical subaxial ${ }^{4}$

Neste trabalho, apresentamos casuística de seis pacientes submetidos a fixação com parafuso de massa lateral em C1, suas indicações e resultados.

\section{MÉTODOS}

Realizou-se avaliação retrospectiva dos pacientes operados por três cirurgiões em conjunto em três diferentes hospitais. Todos pacientes consentiram com a participação no estudo após consulta de seguimento. Foram levantados os seguintes dados: sexo, idade, indicação do procedimento, cirurgia efetuada, exame neurológico pré e pós operatório. Complicações e exames radiológicos pré e pós operatório são apresentados.

\section{TÉCNICA CIRÚRGICA}

A técnica cirúrgica e a anatomia é descrita detalhadamente em outros trabalhos ${ }^{5-10}$. O paciente é colocado em posição prona neutra com fixador de crânio e com uso de extensores da posição semi-sentada para evitar artefatos radiológicos com a utilização de radioscopia, uma vez que a maior parte dos fixadores de crânio disponíveis são radioopacos ${ }^{6}$. O paciente é mantido com a cavidade oral aberta com o uso de uma compressa para avaliação radiológica em incidência antero-posterior.

Uma incisão mediana é realizada, do ínion até cerca do nível da terceira vértebra cervical (ou inferiormente se necessário). Após rebatimento do plano muscular e dissecção subperiostal, expõe-se a lâmina, o processo articular de C2 e o arco posterior de C1, evitando-se a violação de sua região superior. A exposição lateral do arco deve ser cuidadosa devido a hemorragia do plexo venoso vertebral, preferencialmente com lupas e bipolar. Hemostáticos são úteis nesta fase do procedimento. Após, efetua-se a remoção do ligamento atlantoaxial posterior, expondo a raiz posterior de C2. Lentamente, expõe-se a massa lateral de C1, sendo possível sua visualização e palpação dos limites medial e lateral, estipulando-se como ponto de entrada o ponto médio da massa (Figura 1).

Em alguns pacientes com difícil dissecção, autores sugerem a secção da raiz de C2.

Com fluoroscopia, o ponto de entrada é drilado, com angulação de $15^{\circ}$ medial orientado em perfil na direção do tubérculo anterior de C1. Fixação bicortical é recomendada, embora acarrete maiores riscos de lesão da carótida e do esôfago.
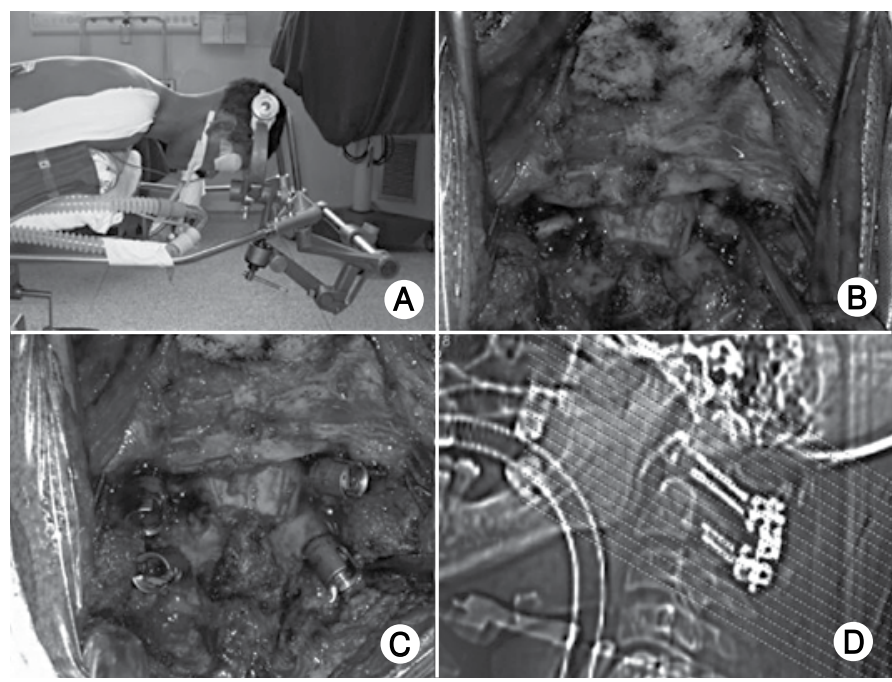

Figura 1. (A) - Paciente posicionado em posição de "sentido" militar, com os extensores para a posição semi-sentada e gaze na cavidade oral. (B) - Visão intraoperatória com deslocamento inferior da raiz de C2 para visualização da massa lateral de C1. (C) - Parafusos, de massa lateral em C1 e de pars em C2, posicionados. (D) - Controle radiográfico da instrumentação.

\section{RESULTADOS}

Foram avaliados seis pacientes operados (quatro homens, duas mulheres) no período de janeiro de 2009 a abril de 2010, submetidos a fixação para artrodese por via posterior da coluna cervical em que foram utilizados parafusos de massa lateral em C1 (Tabela 1).

O tempo médio de seguimento foi de cinco meses (variação de 2 a 10 meses). a causa predominante foi trauma e não houve mortalidade ou morbidade grave nos pacientes avaliados. A fixação com artrodese foi realizada com C2 através parafuso de pedículo em dois casos, parafusos de pars em dois casos e parafusos de lâminas em dois casos. Na porção subaxial da coluna cervical foram utilizados parafusos de massa lateral em dois casos. No primeito, com metástase no processo odontóide de C2, foi necessária extensão da fixação com inclusão das massas laterais de C3-4 para aumentar a força da construção devido a lesão tumoral em C2. No outro paciente, com luxação anterior de C1 devido a rutura traumática de ligamento transverso, devido a fixação insatisfatória na pars de C2 com parafuso relativamente pequeno (12mm), foi optado por estenção da fixação até C3 também para aumentar a

Tabela 1. Pacientes submetidos a fixação com parafusos em massa lateral do atlas.

\begin{tabular}{|c|c|c|c|c|c|c|}
\hline PACIENTE & SEXO & CAUSA DA LESÃO & TIPO DA LESÃO & CIRURGIA & INFECÇÃO & TEMPO DE SEGUIMENTO \\
\hline 1 & M & Trauma & $\begin{array}{l}\text { Fratura de odontóide } \\
\text { tipo II }\end{array}$ & $\begin{array}{l}\text { Parafusos de massa lateral em } \\
\text { C1 e pediculares em C2 }\end{array}$ & Não & 8 meses \\
\hline 2 & M & Trauma & $\begin{array}{l}\text { Fratura do odontóide } \\
\text { tipo II }\end{array}$ & $\begin{array}{c}\text { Parafuso de masa lateral em C1 } \\
\text { e de pars em C2 }\end{array}$ & Não & 4 meses \\
\hline 3 & $\mathrm{~F}$ & Trauma & $\begin{array}{c}\text { Fratura de odontóide } \\
\text { tipo II }\end{array}$ & $\begin{array}{c}\text { Parafuso de massa lateral em } \\
\text { C1, de pars em C2 e massa } \\
\text { lateral em C3 }\end{array}$ & $\begin{array}{l}\text { Infecção superficial } \\
\text { no local de retirada de } \\
\text { enxerto de crista ilíaca }\end{array}$ & 4 meses \\
\hline 4 & M & Trauma & $\begin{array}{l}\text { Lesão do ligamento } \\
\text { transverso do atlas }\end{array}$ & $\begin{array}{c}\text { Parafuso de massa lateral em C1 } \\
\text { de lâmina em C2 }\end{array}$ & Não & 5 meses \\
\hline 5 & $M$ & $\begin{array}{l}\text { Metástase de } \\
\text { próstata }\end{array}$ & $\begin{array}{l}\text { Fratura patologica } \\
\text { do odontóide }\end{array}$ & $\begin{array}{c}\text { Parafuso de massa lateral em } \\
\text { C1, pedículo em C2 e massa } \\
\text { lateral em C3 e C4 }\end{array}$ & Não & 5 meses \\
\hline 6 & $\mathrm{~F}$ & Trauma & $\begin{array}{l}\text { Lesão do ligamento } \\
\text { transverso do atlas }\end{array}$ & $\begin{array}{l}\text { Parafuso de massa lateral em } \\
\text { C1 e de lâmina em C2 }\end{array}$ & Não & 4 meses \\
\hline
\end{tabular}


segurança da fixação. Todos os pacientes se mantiveram neurologicamente intactos, ASIA E no pré e no pós operatório. O paciente cinco apresentava intensa dor cervical axial com melhora imediata após a artrodese e estabilização da fratura.

Em dois casos, onde havia luxação anterior de $\mathrm{C} 1$, a raiz de C2 foi seccionada para facilitar a visualização de massa lateral de C1. Os pacientes evoluíram com parestesia e hipoestesia transitória sem necessidade de tratamento medicamentoso. Por outro lado, dois pacientes, em que as raízes de C2 foram preservadas, evoluíram com dor e parestesia occipital que foi tratada com gabapentina com bom controle dos sintomas.

Em todos os pacientes foi utilizado enxerto de crista ilíaca, ocorrendo apenas um caso de infecção superficial no local da retirada do enxerto, sem necessidade de internação. É importante destacar que não avaliamos a taxa de artrodese durante o seguimento radiológico dos pacientes, embora todos tiveram melhora clínica importante.

\section{DISCUSSÃO}

Muitas técnicas de fixação da região C1-2 foram descritas na literatura até este momento ${ }^{5-7}$. Porém, em muitas circunstâncias, elas não podem ser aplicadas. Por exemplo, o uso de cabos não pode ser feito em pacientes com fraturas no arco posterior de C1 ou em doenças que requeiram a descompressão posterior. O uso de parafusos transarticulares em C1-2 é contra-indicado em cerca de $20 \%$ dos casos devido a localização medial da artéria vertebral ${ }^{11}$. A adequada fixação de C1-2 evita por vezes a necessidade de extensão da fixação na região occipito-cervical, diminuindo muito a morbidade da cirúrgia, especialmente em traumatismos que não cursem com instabilidade occipito-cervical ${ }^{12-14}$.

Diversas variações anatômicas podem acometer C2, assim o cirurgião deve estar também familiarizado com parafusos pediculares, de pars e de lâminas e ser versátil em sua utilização. Nos casos descritos foi necessária a utilização destas três técnicas. Via de regra, pacientes com pedículos adequados (de cerca de quatro ou mais mm e sem vertebral medianizada), optamos por fixação pedicular. Quando está não é possível, usamos parafusos de pars articulares ou de lâminas, conforme dimensões de ambas avaliadas caso a caso com tomografia pré operatória.

Em nossa casuística, nenhum paciente teve complicação sistêmica ou neurológica grave com o procedimento, salvo a frequência de parestesias na região occipital em dois pacientes, com bom controle com gabapentina. A secção da raiz de C2 distal ao gânglio nos pareceu diminuir esta complicação, ao contrário do que esperávamos, sendo realizada em dois casos. Ambos pacientes não tinham queixas de dor nem de parestesias na região no período pós-operatório ${ }^{5}$.

\section{CONCLUSÃO}

O uso de parafusos de massa lateral em C1 para fixação da coluna cervical superior é uma técnica de baixa morbidade e excelentes resultados. O conhecimento adequado da anatomia desta região, bem como uma correta avaliação radiológica individualizada do paciente no pré operatório são os principais elementos para o sucesso desta técnica cirúrgica.

\section{REFERÊNCIAS}

1. Rhoton AL. The foramen magnum. Neurosurgery. 2000;47(Suppl 3):S155-S93.

2. Penning $L$, Wilmink JT. Rotation of the cervical spine. ACT study in normal subjects. Spine (Phila Pa 1976). 1987;12(8):732-8.

3. Goel A, Laheri VK. Plate and screw fixation for atlanto-axial subluxation. Acta Neurochir (Wien). 1994;129(1-2):47-53.

4. Menendez JA, Wright NM. Techniques of posterior C1-C2 stabilization. Neurosurgery, 2007:60(1 Supp1 1):S103-11.

5. Joaquim AF, Ghizoni E, Rubino PA, Anderle DV, Tedeschi $H$, Rhoton AL, Oliveira E. Lateral mass screws of the atlas: surgical technique and anatomy. World Neuro surgery. 2010;74(2):359-62.

6. Mudo ML, Amantéa AV, Cavalheiro S, Joaquim AF. Intraoperative radiological visualization of the occipito-cervical transition and upper cervical spine: technical note Coluna/ Columna, 2009;8(2):197-9.

7. Harms J, Melcher RP. Posterior C1-C2 fusion with polyaxial screw and rod fixation Spine (Phila Pa 1976). 2001 Nov 15;26(22):2467-71.
8. Fiore AJ, Mummaneni PV, Haid RW, Rodts GE, Sasso RC. C1 Lateral Mass Screws: Surgical Nuances Tech Orthop, 2003;17(3):272-7.

9. Seal C, Zarro C, Gelb D, Ludwig S. C1 lateral mass anatomy: Proper placement of lateral mass screws. J Spinal Disord Tech. 2009:22(7):516-23.

10. Rocha R, Safavi-Abbasi S, Reis C, Theodore N, Bambakidis N, de Oliveira E, et al. Working area, safety zones, and angles of approach for posterior C-1 lateral mass screw placement: a quantitative anatomical and morphometric evaluation. J Neurosurg. Spine, 2007;6(3):247-54.

11. Paramore CG, Dickman CA, Sonntag VK. The anatomical suitability of the C1-2 complex for transarticular screw fixation. J Neurosurg, 1996;85(2):221-4

12. Joaquim AF, Patel AP. Occipito cervical trauma: evaluation, classification and treatment Contemp Spine Surg. 2010;11(4):1-5.

13. Joaquim $A F$, Patel AP. C1 and C2 spine trauma: evaluation, classification and treatment. Contemp Spine Surg. 201011(3):1-7;

14. Joaquim AF, Patel AP. Occipito cervical trauma: Evaluation, Classification and Treatment. Contemp Neurosurg. 2010;32(12):1-6. 